\title{
Clinical utility of diagnostic biomarkers in malignant pleural mesothelioma: a systematic review and meta-analysis
}

\author{
Eline Schillebeeckx ${ }^{1,2}$, Jan P. van Meerbeeck (1) ${ }^{1,2,3,4,5}$ and Kevin Lamote $\mathbb{1}^{1,2,3}$
}

${ }^{1}$ Laboratory of Experimental Medicine and Pediatrics, University of Antwerp, Wilrijk, Belgium. ${ }^{2}$ Infla-Med Centre of Excellence, University of Antwerp, Wilrijk, Belgium. ${ }^{3}$ Dept of Internal Medicine, Ghent University, Ghent, Belgium. ${ }^{4}$ Pulmonology and Thoracic Oncology, Antwerp University Hospital, Edegem, Belgium. ${ }^{5}$ European Reference Network for rare respiratory diseases (ERN-LUNG), Frankfurt Am Main, Germany.

Corresponding author: Eline Schillebeeckx (eline.schillebeeckx@uantwerpen.be)

\begin{abstract}
Shareable abstract (@ERSpublications)
Diagnostic biomarkers for malignant pleural mesothelioma seem promising; however, further research is necessary to prove their clinical value. This review provides a thorough overview of the different markers and compares them in several matrices. https://bit.ly/35ni6UO
\end{abstract}

Cite this article as: Schillebeeckx E, van Meerbeeck JP, Lamote K. Clinical utility of diagnostic biomarkers in malignant pleural mesothelioma: a systematic review and meta-analysis. Eur Respir Rev 2021; 30: 210057 [DOI: 10.1183/16000617.0057-2021].

Copyright @The authors 2021

This version is distributed under the terms of the Creative Commons Attribution Non-Commercial Licence 4.0. For commercial reproduction rights and permissions contact permissions@ersnet.org

This article has supplementary material available from err.ersjournals.com

Received: 3 March 2021 Accepted: 8 June 2021

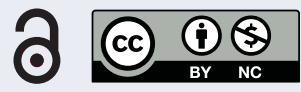

\section{Abstract}

Malignant pleural mesothelioma (MPM) is characterised by late-stage diagnosis and poor prognosis. Currently, no screening tool is advocated and diagnosis is based on invasive techniques, which are not well tolerated. Non-invasive diagnostic biomarkers have shown potential and could have a huge clinical benefit. However, despite extensive research, there is no consensus yet on their clinical use, with many articles reporting contradicting results, limiting their clinical implementation. The aim of this systematic review is therefore to explore the different semi- and non-invasive diagnostic markers in several human matrices and identify those that might clinically be relevant. A total of 100 articles were selected through Web of Science and PubMed, with 56 articles included in the quantitative analysis. Although many studies have reported on the diagnostic accuracy of MPM biomarkers such as serum mesothelin and high-mobility group box protein 1 and plasma fibulin-3, none have resulted in a validated test for early detection. Future research should focus on external validation, combinations into biomarker panels, the inclusion of early stage MPM patients and a combination of different biomarker matrices, as well as new markers.

\section{Introduction}

Malignant pleural mesothelioma (MPM) is a cancer caused by historical exposure to asbestos fibres [1]. MPM has a poor prognosis, with a median survival of 9-12 months after diagnosis and <5\% 5-year survival rate [2]. There are three histological subtypes of MPM, which are prognostic of patient outcome: epithelioid (best outcome), sarcomatoid (worst outcome) and biphasic. MPM carcinogenesis is a multistep process caused by the deep penetration of asbestos fibres into the lung epithelium and the pleural space where they cause mutations by a repeated cycle of pleural irritation, damage and repair, leading to frustrated phagocytosis and the release of pro-oncogenic proteins and growth factors [3, 4]. This multistep process takes time, which is reflected by a long latency period of 30 to 50 years between first asbestos exposure and MPM diagnosis [5]. The clinical symptoms of MPM are usually insidious and nonspecific, and enclose dyspnoea, cough and/or chest pain [1,3]. Hence, a late diagnosis is characteristic for MPM, leading to a dismal prognosis. The gold standard for diagnosing MPM remains histological assessment of thoracoscopically obtained biopsies [1]. However, due to its invasiveness, it is not suitable for each and every patient. Furthermore, treatment of MPM is mostly palliative and consists of a backbone of chemotherapy, with recent promising advancement in immunotherapy (nivolumab and ipilimumab) [1, 3, 6] Hence, research into less invasive biomarkers that could play a role in earlier diagnosis are thriving.

Liquid biomarkers have sparked a lot of interest due to their advantages over a single tissue biopsy. Liquid biomarkers are found in different body fluids, such as serum, plasma, pleural effusions (PEs), exhaled 
breath condensate, saliva and urine and contain a lot of information about the tumour, also at distinct metastatic sites, are less invasive and are easy to obtain [7, 8]. Especially when tissue sampling is hampered by the location of the tumour, liquid biomarkers can be beneficial. Furthermore, they hold the promise of diagnosing diseases at an early stage, even before detection by clinical imaging techniques [7]. The aim of this systematic review is to review the state of the art regarding diagnostic biomarkers for the detection of MPM in different body matrices.

\section{Methods}

\section{Search strategy}

This systematic review was conducted in accordance with the Preferred Reporting Items for Systematic Reviews and Meta-Analyses guidelines [9]. Both PubMed and Web of Science were used as online databases. All articles published before 31 December 2019 were up for review. Keywords were determined based upon the population, exposure, comparison, outcome strategy (supplementary table S1), and their combination was used to detect relevant literature in the aforementioned databases (supplementary table S2).

Only articles published in English assessing the diagnostic accuracy of a biomarker in a human liquid matrix for the diagnosis of MPM were included. Editorial material, letters, reviews, case reports and meta-analyses were excluded. No limitation on the year of publication was imposed and all duplicates were removed. Firstly, the article titles and abstracts were screened by two independent reviewers, after which the full text of the remaining articles was evaluated. Study details (title, author, date), research question, matrix, method, patient population and control group, biomarker, potential confounders, reference test, patient selection, cut-off value, sensitivity, specificity and area under the receiver operating characteristics curve $\left(\mathrm{AUC}_{\mathrm{ROC}}\right)$ were collected. The data was grouped according to the matrix and type of biomarker.

\section{Study quality scoring}

The quality of the articles was assessed using the Quality Assessment of Diagnostic Accuracy Studies 2 scoring system based on the scoring of four key domains: patient selection, index test, reference standard and flow and timing [10]. The risk of bias was determined based on all four domains, while for the risk of applicability only the first three were taken into account. For each domain, predefined questions were answered by yes or no, with “yes' indicating a low risk and "no" a high risk. Questions could also be answered with unclear, when the article did not include the applicable information.

\section{Meta-analysis}

All markers studied by at least two research groups were included in the meta-analysis. Studies dividing the population into subpopulations and not reporting the $\mathrm{AUC}_{\mathrm{ROC}}$ or its (correct) confidence interval, were excluded. When one study reported the diagnostic accuracy of MPM compared to different control groups, the comparison per control group was included. Forest plots were created using the metafor package in R [11].

\section{Results}

A total of 2385 articles matched our search terms in Web of Science and PubMed (Supplementary figure S1). After duplicate removal, 2141 articles remained, which was further reduced to 469 after selection on title and abstract. 100 articles were included in the qualitative analysis after full-text assessment and their characteristics are summarised in supplementary table S3. The majority of the articles reported on serum biomarkers and only mesothelin was studied in all matrices (Supplementary figure S2). 56 studies were included in the quantitative analysis. Interesting markers for which a meta-analysis was not possible are described in the online data supplement.

\section{Quality assessment}

Supplementary figure S3 shows the quality of the 100 included articles. Overall, there are few concerns regarding applicability. All studies included MPM patients; however, there is a high variability in the type of control subjects, ranging from healthy controls with (AE) and without (HC) proven asbestos exposure or those with asbestos-related benign diseases to patients with non-asbestos-related malignancies and benign diseases. Furthermore, some studies only included epithelioid-type MPM patients, which can lead to bias as they tend to lead to a better outcome [12-17]. The most commonly used reference standard was histological or cytological assessment of pleural biopsies, often confirmed by experienced pathologists. However, some articles failed to mention exactly how MPM was diagnosed. The risk of bias does call for some concern. Firstly, some studies excluded patients based on suspicion of infection [18-23], previous therapy for other malignancies [24], other benign or malignant diseases [12, 13, 16, 25-33], mixed and sarcomatoid mesothelioma [13], or unclear diagnosis [34-37], which can lead to a more positive evaluation of the diagnostic capacities of the biomarker. Most studies only included untreated patients. However, this was not seen as an inappropriate exclusion and did not lead to the selection of solely early 
stage MPM. All studies used a cross-sectional, case-control design. Secondly, the cut-off value was often calculated and not pre-specified, introducing a potential bias through the index test. Furthermore, only a few studies mentioned the blinding of the reference test results, while interpreting the index test. Thirdly, some studies failed to mention the used reference standard or reported a combination of different methods, introducing a potential bias. Lastly, as almost all studies only included treatment-naïve patients, there was minimal concern for long intervals between the reference and index test.

\section{Serum biomarkers}

$65 \%$ of all studies report on biomarkers in serum, making it the most investigated matrix.

\section{Proteins}

Mesothelin is a $40 \mathrm{kDA}$ cell surface glycoprotein with low expression in normal mesothelial cells [31]. Together with megakaryocyte potentiating factor (MPF), mesothelin is derived from a $70 \mathrm{kDa}$ precursor [38]. Although mesothelin is normally cell bound, different isoforms can be released into the bloodstream as a result of abnormal splicing or enzymatic cleavage. In the literature, both (soluble) mesothelin and soluble mesothelin-related peptides are often used interchangeably. Enzyme-linked immunosorbent assay (ELISA) was the preferred analytical method, with most studies having used the MesoMark assay. 27 studies were included in the meta-analysis of serum mesothelin (figure 1a) [13, 20, 21, 29, 35, 39-61]. All studies reported an overexpression in MPM patients compared to different control groups, with cut-off values ranging between $0.55 \mathrm{nM}$ and $2.4 \mathrm{nM}$. When looking at the different $\mathrm{AUC}_{\mathrm{ROC}}$ values, it is important to not lose track of the control group that was used. The highest $A_{U} C_{R O C}$ was obtained when comparing to a limited and less representative control population consisting of only benign PEs $\left(\mathrm{AUC}_{\mathrm{ROC}}=0.93 ; \mathrm{AUC}_{\mathrm{ROC}}=0.94\right)$ [35, 57]. When only stage I MPM patients were compared to AE controls, an $\mathrm{AUC}_{\mathrm{ROC}}$ of 0.74 was reported, indicating a potential for early diagnosis [47]. However, it was significantly lower than the $A U C_{R O C}$ when all stages of MPM were included $\left(A U C_{R O C}=0.81\right.$ ), in line with the intuitive idea that early stage diagnosis is more challenging. The specificity of serum mesothelin is questionable as a lower AUC was obtained when compared to other cancer types and metastases. A higher serum mesothelin concentration in the epithelioid subtype compared to other subtypes was explicitly described by most studies [20, 39-41, 47, 59]. The articles that could not find a significant difference between the two subtypes, did find a trend towards higher levels in the epithelioid subtype with the lack of significance most likely due to the limited amount of included sarcomatoid patients [42, 47, 61].

Only four studies investigated MPF, which all reported an elevation in MPM patients [34, 50, 56, 59]. In a large population (85 MPM patients and 275 controls), a high correlation between MPF and mesothelin was reported [59]. However, due to a lack in sensitivity (67.4\% when compared to benign pleural disease) and specificity (60.8\% when compared to metastases), in general, mesothelin seems to be more performant than MPF in serum (AUC ${ }_{\mathrm{ROC}}=0.81$ and 0.78 , respectively) (figure $1 \mathrm{~b}$ ) [34, 50, 56, 59]. A significant link between higher MPF levels and the epithelioid subtype as well as the association with advanced stage was found $[34,59]$.

Osteopontin (OPN) is an integrin-binding protein involved in cell-matrix interactions [38]. The reported cut-off values vary tremendously, ranging from $16.06 \mathrm{ng} \cdot \mathrm{mL}^{-1}$ to $139.1 \mathrm{ng} \cdot \mathrm{mL}^{-1}$. Studies focussing on differential diagnosis with benign PEs performed very poorly, with $\mathrm{AUC}_{\mathrm{ROC}}$ values no better than random (figure 1c) $[34,49]$. Studies including $\mathrm{AE}$ and $\mathrm{HC}$ individuals as controls performed remarkably better $\left(\mathrm{AUC}_{\mathrm{ROC}}=0.89\right.$ and 0.86, respectively) [31, 62]. All studies reported a higher level of OPN in MPM patients compared to controls. However, two studies reported no difference in OPN levels when compared to other diseases $[34,50]$. These results stress that serum OPN is not optimal for differential diagnosis as it lacks specificity.

Integrin-linked kinase (ILK) is an intracellular kinase linking $\beta$-integrins to the cytoskeleton. It plays a role in chronic inflammatory processes and its overexpression can induce an epithelial-mesenchymal transition and thus a tumorigenic phenotype in epithelial cells [63]. Two different diagnostic outcomes were reported, with a $A U C_{R O C}$ values of 0.69 and 0.89 (figure 1d) [63, 64]. The lower diagnostic accuracy was obtained when comparing to AE controls, while a better outcome was reported with malignant and benign chest diseases. Whether this difference can be explained by the use of different methods (western blot or ELISA) or a lower performance against asbestos exposure/related diseases is currently unclear. However, both studies were conducted by the same research group without explicitly describing their reference test, urging further research.

Thioredoxin-1 (TRX) is a protein with antioxidant properties as it can mobilise the antioxidant defence mechanism decreasing reactive oxygen species [29]. In MPM patients, TRX levels are increased due to the 

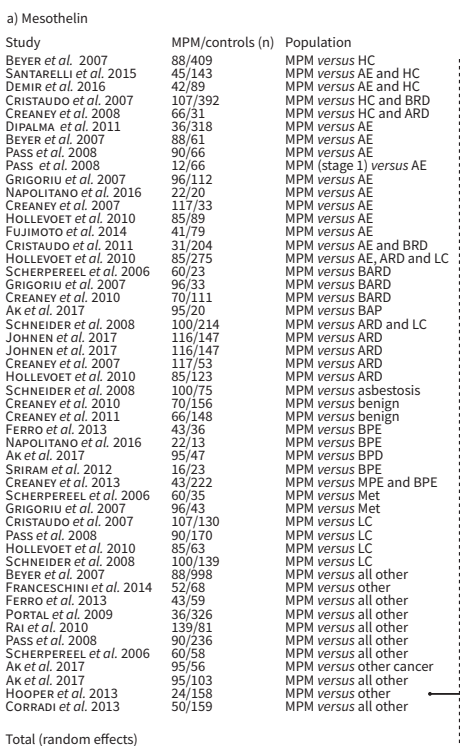

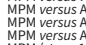

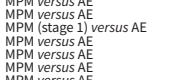

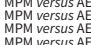

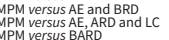

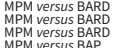
MPM versus BARD
MPM versus
MPM Vers
MPM

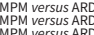
MPM versus ARD
MPM versLARD
MPM versus MPM versus asbestosis
MPM versus benign
MPM versus benign

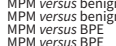
$M P M$ versus $B P E$
$M P M$ versus BPD
MPM versus BPE
MPM versus MPE and BPE MPM versus B
MPM versus MPE
MPM versus Met MPM versus Met
MPM versus Me
MPPM versus $L C$
MPP versus LC MPP verssus $L C$
$M P M$ versus $L C$
MPM versus LC MPM versus $L C$
MPM versus all other
MPM versus other
MPM versus all other MPM versus other
MPM versus all other
MPM versus all other
MPM versus all other MPM versus all other
MPM versus all other
MPM versus all other MPM versus other can
MPM versus all other
MPM versus other MPM verssus other

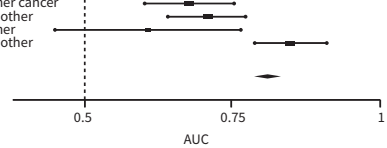

b) MBF
Study
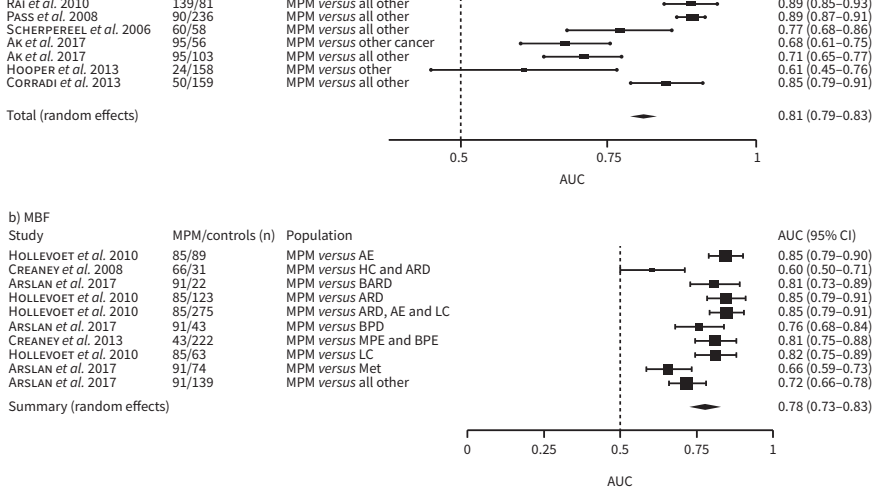

$0.81(0.79-0.83)$ c) OPN

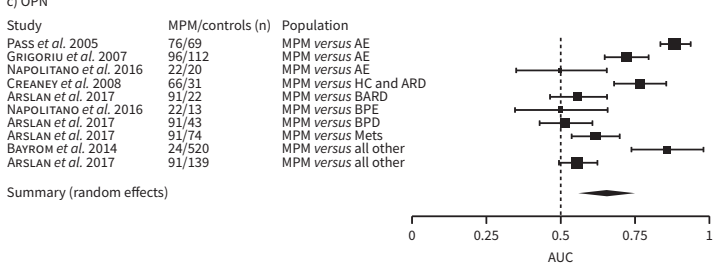

d) ILK
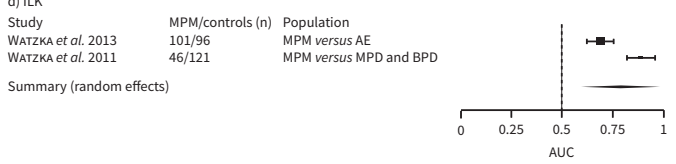

e) TRX

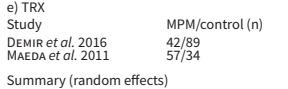

Population
MPM versus AE and HC
MPM versus AE and BARD

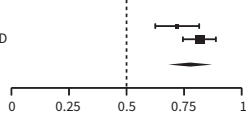

AUC $(95 \%$ Cl) $0.72(0.63-0.81)$ $0.77(0.68-0.87)$

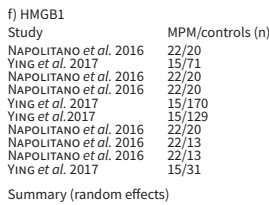
Summary (random effe
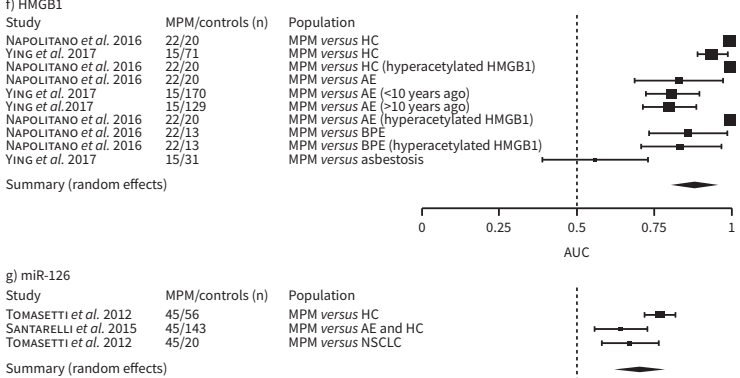
UC $(95 \% \mathrm{Cl}$ $.00(1.00-1.00$
$0.94(0.89-0.99$ -1.000
0 $.81(0.73-0.89$
.80
$0.72-0.88$

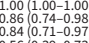
$0.56(0.39-0.73)$
$0.88(0.80-0.93)$

Summary (random effects)

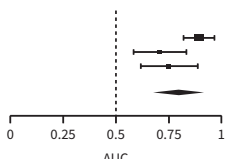

$\mathrm{AUC}(95 \% \mathrm{C})$ $0.89(0.82-0.97$
$0.71(10.58-0.84$
$0.75(0.62-0.89$ $0.80(0.68-0.91)$

FIGURE 1 Forest plot for the area under the curve (AUC) of serum markers in distinguishing malignant pleural mesothelioma (MPM) from different controls. AE: asbestos exposed; ARD: asbestos-related disease; BAP: benign asbestos pleurisy, BARD: benign asbestos-related disease; BPD: benign pleural disease; BPE: benign pleural effusion; BRD: benign respiratory disease; HC: healthy control; HMGB1: high mobility group box protein 1; ILK: integrin-linked protein kinase; LC: lung cancer; Met: metastasis; miR: microRNA; MPF: megakaryocyte potentiating factor; MPD: malignant pleural disease; MPE: malignant pleural effusion; NSCLC: non-small cell lung cancer; OPN: osteopontin; TRX: thioredoxin-1. References in panel a): AK et al. 2017 [51]; Beyer et al. 2007 [42]; Cristaudo et al. 2007 [40]; Creaney et al. 2008 [50]; Creaney et al. 2010 [52]; Creaney et al. 2007 [54]; CReaney et al. 2011 [55]; Creaney et al. 2013 [56]; Creaney et al. 2007 [61]; Corradi et al. 2013 [58]; Demir et al. 2016 [29]; Dipalma et al. [39]; Ferro et al. 2013 [44]; Fujimoto et al. 2014 [60]; Franceschini et al. 2014 [43]; Grigoriu et al. 2007 [21]; Hollevoet et al. 2010 [59]; Hooper et al. 2013 [35]; Johnen et al. 2007 [53]; Napolitano et al. 2016 [49]; Pass et al. 2008 [47]; Portal et al. 2008 [45]; Ral et al. 2010 [46]; Santarelli et al. 2015 [48]; Scherpereel et al. 2006 [20]; SRiram et al. 2012 [57]. Panel b): Arslan et al. 2017 [34]; Creaney et al. 2008 [50]; Creaney et al. 2013 [56]; Hollevoet et al. 2010 [59]. Panel c): Arslan et al. 2017 [34]; Bayram et al. 2014 [31]; Pass et al. 2005 [62]; Creaney et al. 2008 [50]; Grigoriu et al. 2007 [21]; Napolitano et al. 2016 [49]. Panel d): Watzka et al. 2011 [63]; Watzka et al. 2013 [64]. Panel e): Demir et al. 2016 [29]; Maeda et al. 2011 [65]. Panel f): Napolitano et al. 2016 [49]; Ying et al. 2017 [66]. Panel g): Santarelli et al. 2015 [48]; Tomasetti et al. 2012 [19].

heightened antioxidant capacity (figure 1e) [29, 65]. The median TRX concentrations varied, with the higher concentrations linked to early stage MPM patients (stage I and II). Nevertheless, this did not result in an overall higher discriminatory capacity. At a cut-off of $156.67 \mathrm{ng} \cdot \mathrm{mL}^{-1}$, a sensitivity of $92.9 \%$ (at $77.6 \%$ specificity) was obtained, which might render it interesting for screening purposes. As no comparisons were made with other cancer types, TRX’s capacities as a discriminatory marker remain unknown.

High-mobility group box protein 1 (HMGB1) is a damage-associated molecular pattern protein that is released in the extracellular space during necrosis [49]. When HMGB1 resides in the nucleus, it is mainly non-acetylated, while the cytoplasmic hyperacetylated HMGB1 is secreted into the extracellular space where it attracts macrophages leading to a chronic inflammatory state. Therefore, it is hypothesised that the non-hyperacetylated form is present is AE individuals, while the hyperacetylated form is actively released during carcinogenesis. Overall, HMGB1 is a very promising biomarker, with the hyperacetylated form performing slightly better (figure 1f) [49]. However, the superiority of hyperacetylated HMGB1 should be further investigated. When MPM patients were compared to asbestosis patients, no differences were found. Furthermore, HMGB1 was reported to be an excellent marker for distinguishing asbestosis patients from HC and AE [66]. Unfortunately, as no comparisons with other malignancies are available yet, the inability 
to discriminate between MPM and asbestosis might indicate a lack in overall specificity. These results should be interpreted with caution, as only two studies with limited sample sizes were included in the meta-analysis, urging the need for future validation.

MiRNA

MicroRNA (miRNA, miR) stands for non-coding RNA that has an important role in regulating gene expression. Studies focussing on miRNA all reported an increased expression of miR-197-3p, miR-1281, miR548-3p, miR-20a, miR-625-3p, has-1,3 miR-2053 and miR-34b/c. Only miR-126 was downregulated, which might be explained by its potential tumour suppressor function [18, 19]. With a combined $A U C_{R O C}$ of 0.80 , miR-126 is one of the best performing serum markers (figure 1g). However, the best result stems from the comparison with solely $\mathrm{HC}$, possibly resulting in an overoptimistic summary $\mathrm{AUC}_{\mathrm{ROC}}$. Furthermore, the lack in specificity (54\%) when compared to $\mathrm{HC}$ and AE reveals a suboptimal discriminatory capacity when asbestos exposure is included in the control group.

Biomarker panels

When serum OPN and mesothelin were measured in the same individual, a positive correlation with disease outcome was found comparing MPM patients to patients with pleural plaques, AE or HC [21, 31]. However, this was contradicted when combining both markers did not lead to an improvement in diagnostic accuracy $[21,46]$. Furthermore, neither the addition of OPN nor of MPF had any added value over mesothelin on its own $[50,59]$. As mesothelin and MPF both stem from the same precursor, it is not surprising that no significant difference in diagnostic accuracy between the two was found. Lastly, CA125 is a transmembrane mucin and known tumour marker [61]. In over 50\% of cases, CA125 and mesothelin levels were not linked, which might lead to an additive effect when combined in a panel. Unfortunately, CA125 did not improve the sensitivity of mesothelin in detecting MPM. In contrast, carcinoembryonic antigen (CEA), chitinase-like protein (YKL-40) and cytokeratin 19 fragment (CYFRA-21) did improve the diagnostic performance of mesothelin [25, 30, 58].

\section{Plasma biomarkers}

Of the 19 studies looking into plasma biomarkers, 11 investigated mesothelin, two calretinin, one thrombomodulin, six OPN, three fibulin-3, five miRNA and one other (8-hydroxy-2'-deoxyguanosine, platelet-derived growth factor-h, hepatocyte growth factor, basic fibroblast growth factor and vascular endothelial growth factor-h, matrix metalloproteinase (MMP) 2, MMP9, tissue inhibitor of metalloproteinase (TIMP) 1 and TIMP2). ELISA was the preferred analytical method for plasma proteins.

\section{Proteins}

OPN is the most investigated plasma protein, with five studies included in the meta-analysis (figure 2a) $[13,21,22,46,55]$. However, with AUC $_{\text {ROC }}$ values ranging from 0.568 to 0.795 and a summary AUC ROC of 0.69 , it seems to be outperformed by other plasma biomarkers independent of the control group.

Fibulin-3 is a secreted glycoprotein which has shown to promote tumour growth and invasion via phosphorylation of the epidermal growth factor receptor and downstream activation of the AKT (protein kinase B) signalling pathway [67]. The Pass and Jiang groups both reported a high diagnostic potential with $\mathrm{AUC}_{\mathrm{ROC}}$ values ranging from 0.81 when compared to asbestosis patients to 0.92 when compared to HC $[16,68]$. However, a lower accuracy was achieved when discriminating MPM from a control group including patients with benign asbestos-related disease, malignant and non-malignant effusions (AUC=0.671) [67]. The Pass's group also performed a validation analysis for the comparison with AE, where a very low sensitivity of $33 \%\left(\mathrm{AUC}_{\mathrm{ROC}}=0.87\right.$ ) was obtained. This is significantly different from the reported sensitivity of $71.32 \%$ during the discovery phase, further emphasizing the importance of biomarker validation. Overall, the three studies resulted in a summary $\mathrm{AUC}_{\mathrm{ROC}}$ of 0.91 , which is the highest for plasma (figure $2 \mathrm{~b}$ ).

All studies confirmed a higher value of plasma mesothelin in MPM compared to controls [23, 46, 55, 67, 69]. The reported cut-off values ranged from $1.963 \mathrm{nM}$ to $2.5 \mathrm{nM}$. When plasma mesothelin levels of MPM patients were compared to those of patients with benign respiratory diseases, malignant and non-malignant effusions, an $\mathrm{AUC}_{\mathrm{ROC}}$ of 0.816 was obtained, with $56 \%$ sensitivity and $96 \%$ specificity [67]. When $\mathrm{AE}$ was used as a control group, contradictory results were found, with an $\mathrm{AUC}_{\mathrm{ROC}}$ values of 0.66 and 0.93 , respectively [23, 69]. The quantitative analysis resulted in an overall $\mathrm{AUC}_{\mathrm{ROC}}$ of 0.86 (figure 2c).

\section{Biomarker panels}

As there is limited effect of the matrix on mesothelin measurements, plasma is preferred over serum for biomarker discovery due to its better stability. When mesothelin, calretinin and MPF were combined into a 
a) OPN

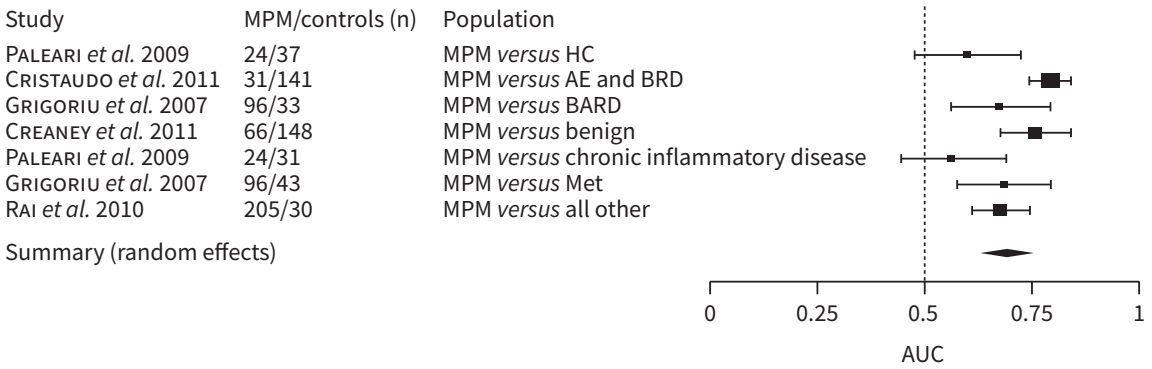

AUC $(95 \% \mathrm{Cl})$

$0.60(0.48-0.73)$

$0.80(0.75-0.84)$

$0.68(0.56-0.79)$

$0.76(0.68-0.84)$

$0.57(0.45-0.69)$

$0.69(0.58-0.80)$

$0.68(0.62-0.74)$

$0.69(0.63-0.75)$

b) Fibulin-3

Study

MPM/controls ( $n$ ) Population

JIANG et al. 2017

JIANG et al. 2017

PASs et al. 2012

JIANG et al. 2017

JIANG et al. 2017

PASs et al. 2012

PAss et al. 2012

PAss et al. 2012

PASs et al. 2012

CREANEY et al. $2014 \quad 82 / 12$

PASs et al. $2012 \quad 92 / 290$

$15 / 94$

$15 / 21$

$92 / 136$

$15 / 74$

$15 / 29$

$92 / 259$

$92 / 8$

MPM versus $\mathrm{HC}$

MPM versus $A E$

MPM versus $A E$

MPM versus PP

MPM versus asbestosis

MPM versus no PE

MPM versus benign $\mathrm{PE}$

$M P M$ versus malignant $P E$

MPM versus PE other cause

MPM versus all other

MPM versus all other

Summary (random effects)

c) Mesothelin

Study

AmATI et al. 2008

JOHNEN et al. 2018

AMATI et al. 2018

CREANEY et al. 2011

CREANeY et al. 2014

RAl et al. 2010

Total (random effects)

$\begin{array}{ll}\text { MPM/controls }(n) & \text { Population } \\ 22 / 54 & \text { MPM versus HC } \\ 24 / 136 & \text { MPM versus AE } \\ 22 / 94 & \text { MPM versus AE } \\ 66 / 148 & \text { MPM versus benign } \\ 82 / 120 & \text { MPM versus non MPM } \\ 139 / 81 & \text { MPM versus all other }\end{array}$

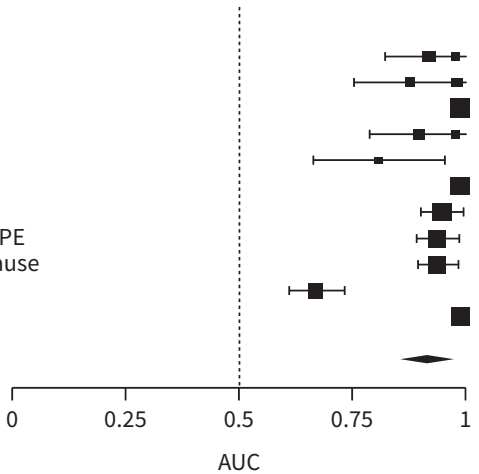

AUC $(95 \% \mathrm{Cl})$

$0.92(0.83-1.00)$

$0.88(0.76-1.00)$

$0.99(0.98-1.00)$

$0.90(0.79-1.00)$

$0.81(0.67-0.95)$

$0.99(0.98-1.00)$

$0.95(0.90-1.00)$

$0.94(0.89-0.99)$

$0.94(0.89-0.99)$

$0.67(0.61-0.73)$

$0.99(0.98-1.00)$

$0.91(0.86-0.97)$

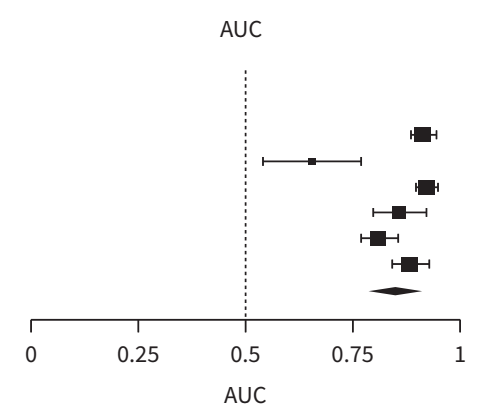

AUC $(95 \% \mathrm{Cl})$

$0.92(0.89-0.95)$

$0.66(0.55-0.77)$

$0.93(0.91-0.95)$

$0.86(0.80-0.93)$

$0.82(0.77-0.86)$

$0.89(0.85-0.93)$

$0.86(0.79-0.92)$

FIGURE 2 Forest plot for the area under the curve (AUC) of plasma markers in distinguishing malignant pleural mesothelioma (MPM) from different controls. AE: asbestos exposed; BARD: benign asbestos related disease; BRD: benign respiratory disease; HC: healthy control; Met: metastasis; PE: pleural effusion; PP: pleural plaques; OPN: osteopontin. References in panel a): Palearl et al. 2009 [22]; CRISTAUdo et al. 2011 [13]; GrigorIU et al. 2007 [21]; Creaney et al. 2011 [55]; Ral et al. 2010 [46]. Panel b): Jiang et al. 2017 [16]; Pass et al. 2012 [68]; Creaney et al. 2014 [67]. Panel c): Amatiet al. 2008 [23]; Johnen et al. 2018 [69]; Creaney et al. 2011 [55]; Creaney et al. 2014 [67]; RAl et al. 2010 [46].

diagnostic biomarker panel, an increase of the AUC to 0.944 was obtained in a male population and 0.937 in a female population. The corresponding sensitivities are $82 \%$ and $87 \%$ at a pre-set specificity of $95 \%$ [70]. The added value of combining mesothelin and calretinin was further confirmed by JoHNEN et al. [69]. Combining mesothelin and miR-103a-3p resulted in higher sensitivity (86\%) and specificity (85\%) [71]. Lastly, although miR132-3p ( UUC $_{\mathrm{ROC}}=0.76$ ) seems less effective in diagnosing MPM compared to mesothelin $\left(\mathrm{AUC}_{\mathrm{ROC}}=0.81\right)$, the combination of both is superior to their individual diagnostic capacities, resulting in an $\mathrm{AUC}_{\mathrm{ROC}}$ of 0.90 [71, 72]. A similar positive effect was seen for the combination of miR-132-3p and miR-126 [72].

\section{PE biomarkers}

In total, $36 \%$ of the included studies investigated biomarkers in PEs (Supplementary table S3). Proteins are the most investigated biomarkers (89\%) followed by DNA (5.5\%) and miRNA (5.5\%).

\section{Proteins}

The diagnostic effectiveness of PE mesothelin is shown in figure 3a [20, 21, 35, 43, 44, 47, 56, 57, 67, 73-82]. All 19 studies reported relatively similar $\mathrm{AUC}_{\mathrm{ROC}}$ values ranging from 0.70 to 0.93 , leading to a 
summary total of 0.83 . The largest study included 128 MPM patients and 1147 controls and obtained a

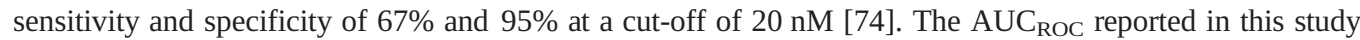
is comparable to the overall calculated $\mathrm{AUC}_{\mathrm{ROC}}$, which might be caused by the higher weight due to its small confidence interval. Similar to the other matrices, all studies reported an elevated level of mesothelin in MPM patients compared to controls. A large heterogeneity was found in reported cut-off values, ranging from $6 \mathrm{nM}$ to $50 \mathrm{nM}$. Both the highest and lowest cut-off values were reported by Aleman et al. [76], who compared a group of 18 MPM patients to 51 controls (including lung cancer patients, patients with other cancers, patients with pneumonia, TB and idiopathic pulmonary disease). Sensitivity and specificity of $72 \%$ and $46 \%$ and $44 \%$ and $100 \%$ were obtained for the lowest and highest cut-off value, respectively. The most commonly used cut-off value in PE lies around 20nM, with sensitivities and specificities ranging from $44 \%$ to $100 \%$ and $46 \%$ to $100 \%$.

Contradictory results were found for fibulin-3. PAss et al. [68] reported higher fibulin-3 concentrations in PE of MPM patients compared to patients with PE (both benign and malignant, related to AE or not), with $\mathrm{AUC}_{\mathrm{ROC}}$ values of 0.91 and 0.95 in two independent cohorts. However, this could not be reproduced, with other $\mathrm{AUC}_{\mathrm{ROC}}$ values ranging from 0.42 to $0.59[67,78]$. With a summary $\mathrm{AUC}_{\mathrm{ROC}}$ of 0.68 , PE fibulin-3 is not a good standalone diagnostic biomarker (figure 3b). Further research is advised due to the heterogenous results, the limited number of studies and the lack of asbestos-related control groups.

A very promising but less investigated marker for MPM, is CYFRA-21-1, which is the soluble fragment of cytokeratin 19. In malignant tissues, CYFRA-21-1 is released into circulation after apoptosis or caspase-3 cleavage. As carcinogenesis can cause apoptosis, it is not surprising that all studies reported an elevated level of CYFRA-21-1 in MPM patients compared to controls [80, 82, 83]. The diagnostic accuracy is not outstanding, with $\mathrm{AUC}_{\mathrm{ROC}}$ values ranging from 0.65 to 0.76 and an overall $\mathrm{AUC}_{\mathrm{ROC}}$ of 0.73 (figure $3 \mathrm{c}$ ). No major differences in accuracy between the different control groups could be detected; however, no benign or asbestos-related conditions were included.
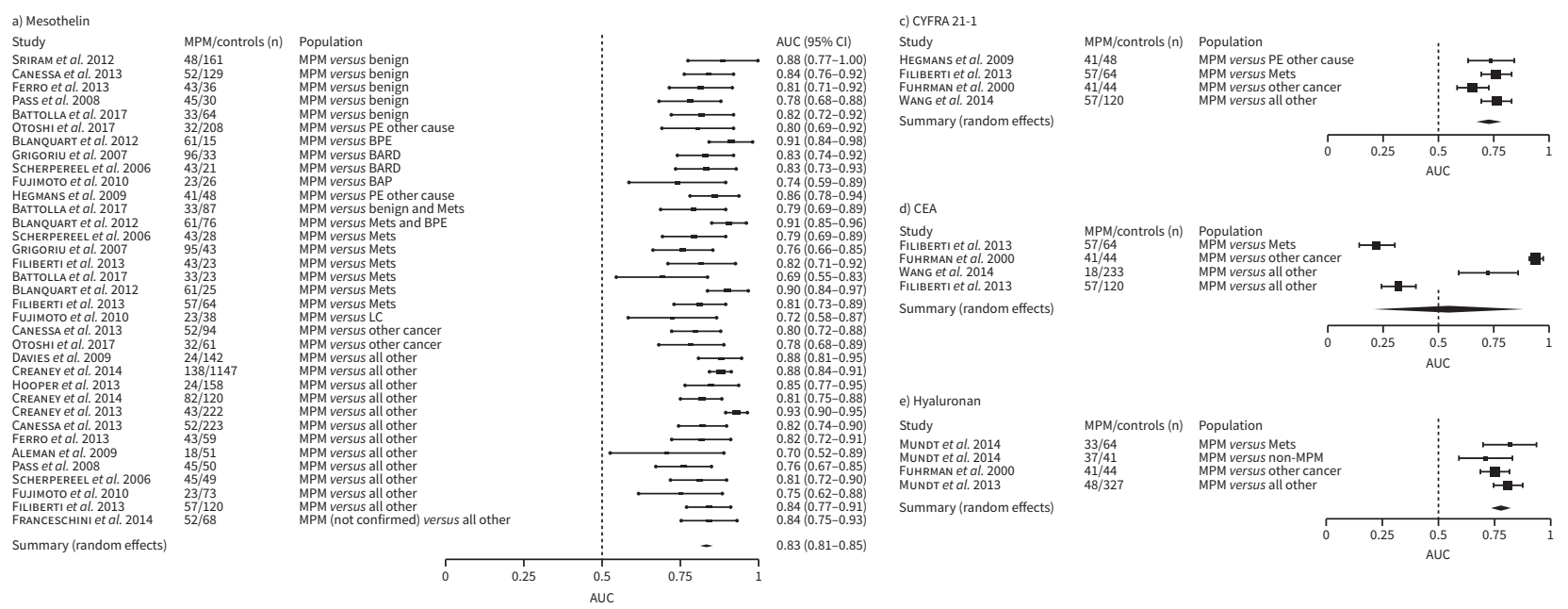

$\operatorname{AUC}(95 \% \mathrm{Cl})$
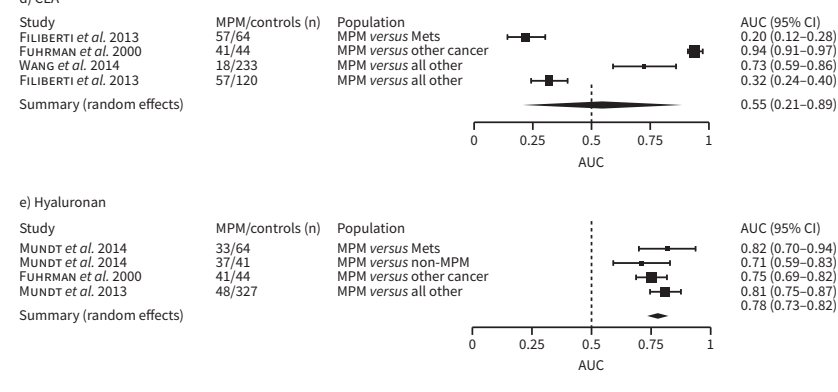

AUC $(95 \%$ (1)

$\operatorname{AUC}(95 \% \mathrm{Cl})$

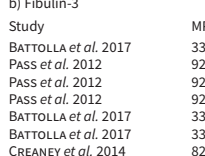

Summary (random effects)
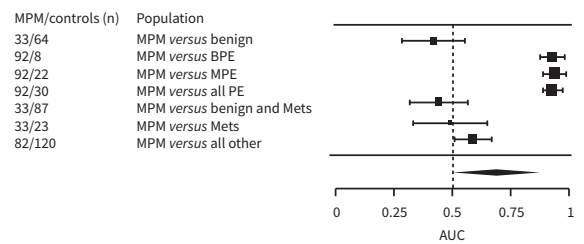

$0.42(0.29-0.55)$

$0.94(0.89-0.99)$

$.93(0.89-0.97)$

$.44(0.32-0.56$
$.49(0.33-0.65)$
$0.59(0.51-0.67)$

$0.59(0.51-0.67)$

$0.68(0.50-0.87)$

FIGURE 3 Forest plot for the area under the curve (AUC) of pleural effusion markers in distinguishing malignant pleural mesothelioma (MPM) from different controls. BARD: benign asbestos related disease; BAP: benign asbestos pleurisy; BPE: benign pleural effusion; CEA: carcinoembryonic antigen; LC: lung cancer; Mets: metastasis; MPE: malignant pleural effusion; PE: pleural effusion. References in panel a): SRIRAM et al. 2012 [57]; Canessa et al. 2013 [75]; Ferro et al. 2013 [44]; PAss et al. 2008 [47]; Battolla et al. 2017 [78]; Otoshi et al. 2017 [81]; Blanquart et al. 2012 [79]; Grigoriu et al. 2007 [21]; Scherpereel et al. 2006 [20]; Fujimoto et al. 2010 [77]; Hegmans et al. 2009 [82]; Filiberti et al. 2013 [80]; Davies et al. 2009 [73]; CReaney et al. 2014 [67]; Hooper et al. 2013 [35]; Aleman et al. 2009 [76]; Franceschini et al. 2014 [43]. Panel b): Battolla et al. 2017 [78]; Pass et al. 2012 [68]; Creaney et al. 2014. Panel c): Hegmans et al. 2009 [82]; Filiberti et al. 2013 [80]; Fuhrman et al. 2000 [83]; Wang et al. 2014 [36]. Panel d): Filiberti et al. 2013 [80]; Fuhrman et al. 2000 [83]; Wang et al. 2014 [36]. Panel e): Mundt et al. 2014 [33]; Fuhrman et al. 2000 [83]; Mundt et al. 2013 [37]. 
CEA is a glycoprotein involved in cell adhesion. In healthy individuals, very low levels of CEA are detectable in the bloodstream. An increase in several cancer types and non-cancerous conditions has been reported. Hence, MPM patients are also expected to have an elevated CEA level. Although two studies indicated this expected increase, one study reported a decrease when compared to other types of cancer $[36,80,83]$. This indicates that CEA is elevated in different cancer types and therefore not specific for MPM. Due to these inconclusive results and an overall $\mathrm{AUC}_{\mathrm{ROC}}$ of 0.55 , no conclusion can be drawn regarding the diagnostic accuracy of PE CEA (figure 3d). Due to its lack of specificity, the use of CEA as a differential diagnostic marker is not advised. Further research including asbestos-exposed individuals should clarify whether CEA might be interesting as a screening marker.

Hyaluronan (HA) or hyaluronic acid is a major compound of the extracellular matrix, regulating cell adhesion and motility, as well as mediating cell proliferation and differentiation [84]. Three studies investigated PE HA, but only two validated their findings in an independent cohort [33, 37]. Only patients with other cancers, benign diseases and metastases were included as controls, so no conclusion can be drawn about HA as an MPM screening biomarker. In PE, HA on its own does not have the best diagnostic accuracy, with an $\mathrm{AUC}_{\mathrm{ROC}}$ of 0.78 (figure 3e).

Biomarker panels

A similar diagnostic accuracy of PE HA and MPF was found [37]. Their combination resulted in a slightly better $\mathrm{AUC}_{\mathrm{ROC}}(0.81$ versus 0.83$)$. A protein biomarker panel that does exceed its individual performance, consists of C-C motif chemokine ligand 2, galectine-3, mesothelin, and secretory leukocyte protease inhibitor [79]. With a sensitivity and specificity of $93.8 \%$ and $64.9 \%$, respectively, the combination of mesothelin and the CYFRA-21-1/CEA ratio is also a strong contender as an MPM biomarker panel [81]. As this combination helped to rule out MPM among the high-risk population, it is a valuable option for screening purposes. The combination of CYFRA-21-1 and CA-15-3 resulted in an elevated sensitivity (100\%) and specificity (83\%) [27]. Furthermore, a combination of CA-15-3, CYFRA-21-1 and CA19-9 resulted in a higher sensitivity, but decreased the specificity [27]. Lastly, the panel of three miRNAs (miR-200c, miR-210 and miR-143) resulted in a summary AUC $_{\mathrm{ROC}}$ of 0.92 when comparing MPM patients to both patients with benign diseases and patients with adenocarcinoma [85].

\section{Urine biomarkers}

Proteins

As urine volume and renal function have effects on protein concentration, urinary mesothelin concentrations were standardised against urinary creatinine concentrations [52]. Again, the level of mesothelin was found to be significantly higher in MPM patients compared to controls. When discriminating MPM patients from those with benign disease, an $\mathrm{AUC}_{\mathrm{ROC}}$ of 0.87 was obtained. A correlation was found with serum mesothelin, but only in the MPM group.

\section{Reported confounding factors}

When looking at diagnostic tests, the risk of confounding factors should always be considered. The main confounding factor seemed to be the histological subtype, with epithelioid tumours being associated with higher diagnostic accuracy of the biomarkers assessed. However, this was contradicted by several studies for serum mesothelin, miRNA, a protein panel, midkine, ILK and the DNA integrity index [28, 41, 47, 51, $57,64,86]$. Weber's group also did not find a difference between different subtypes studying miR-103 in plasma [87]. Secondly, it is not surprising that for most biomarkers the most pronounced differences were found when comparing controls to advanced stages of MPM. In contrast, the influence of body mass index (BMI), smoking, gender and age seemed to have limited effect [16, 23, 29, 35, 63, 68]. However, both BAYRAm et al. [31] and PALEARI et al. [22] reported a significant influence of gender on the serum and plasma OPN levels. Furthermore, a positive relationship was found between smoking status and serum OPN levels [31]. Hollevoet et al. [88] studied the effect of clinical covariates on the diagnostic accuracy of both mesothelin and MPF in serum and reported an increase in accuracy when age decreased or the glomerular filtration rate or BMI increased [88].

\section{Discussion}

Liquid biomarkers can be found in different body fluids, such as serum, plasma, PEs, exhaled breath condensate, saliva, sweat and urine. With their advantage of being minimally invasive, they have a huge benefit over the current golden standard of biopsy-driven diagnosis. Furthermore, a large population of asbestos-exposed individuals do not have access to a readily accessible screening device, which leads to a psychological burden. Being able to relieve people from this stress will have a positive effect on their overall well-being. A rapid and accurate early stage diagnosis can also have a positive effect on the dismal prognosis of MPM, as a potential radical therapy can be initiated earlier. As research for newer and 
curative therapies is booming, diagnostic tools cannot stay behind. Unfortunately, there is still a lot of variability in the reported outcomes of diagnostic biomarker studies. This can mainly be attributed to the control groups included, ranging from only those patients with PEs to patients with benign or malignant conditions and asbestos-related diseases. Due to this heterogeneity, it is difficult to directly compare the results of different individual studies. However, this heterogeneity can be useful to assess the full diagnostic capacity of a certain marker. The inclusion of $\mathrm{HC}$ and $\mathrm{AE}$ as controls is more representative for a screening test, while different types of malignancy and benign asbestos-related diseases can verify whether a differential diagnosis is possible. Furthermore, many studies show that epithelioid mesothelioma is easier to differentiate from controls compared to other MPM histological subtypes. As a result, studies only including the epithelioid subtype can have biased positive results. Both a representative cohort of patients and controls are therefore crucial in future studies. Another point of concern is the cut-off value. As the majority of studies do not have a predefined cut-off value, it leads to a variety of cut-off values, each perfect for the specific cohort used in that study. Unfortunately, many studies disagree on the perfect value, rendering it clinically useless. Therefore, studies including both a verification and a validation cohort or follow-up studies, validating the results in an independent cohort, are necessary.

Many biomarkers have been studied in different matrices. For mesothelin, the highest diagnostic accuracy was detected in plasma, closely followed by PE and serum (figures 2-4). Its levels in PE were significantly higher compared to those in serum, which might be explained by the fact that mesothelin is derived from a cell-bound protein produced locally and released directly into the pleural cavity [44]. Although this was confirmed by most studies, Scherpereel et al. [20] found a significant correlation

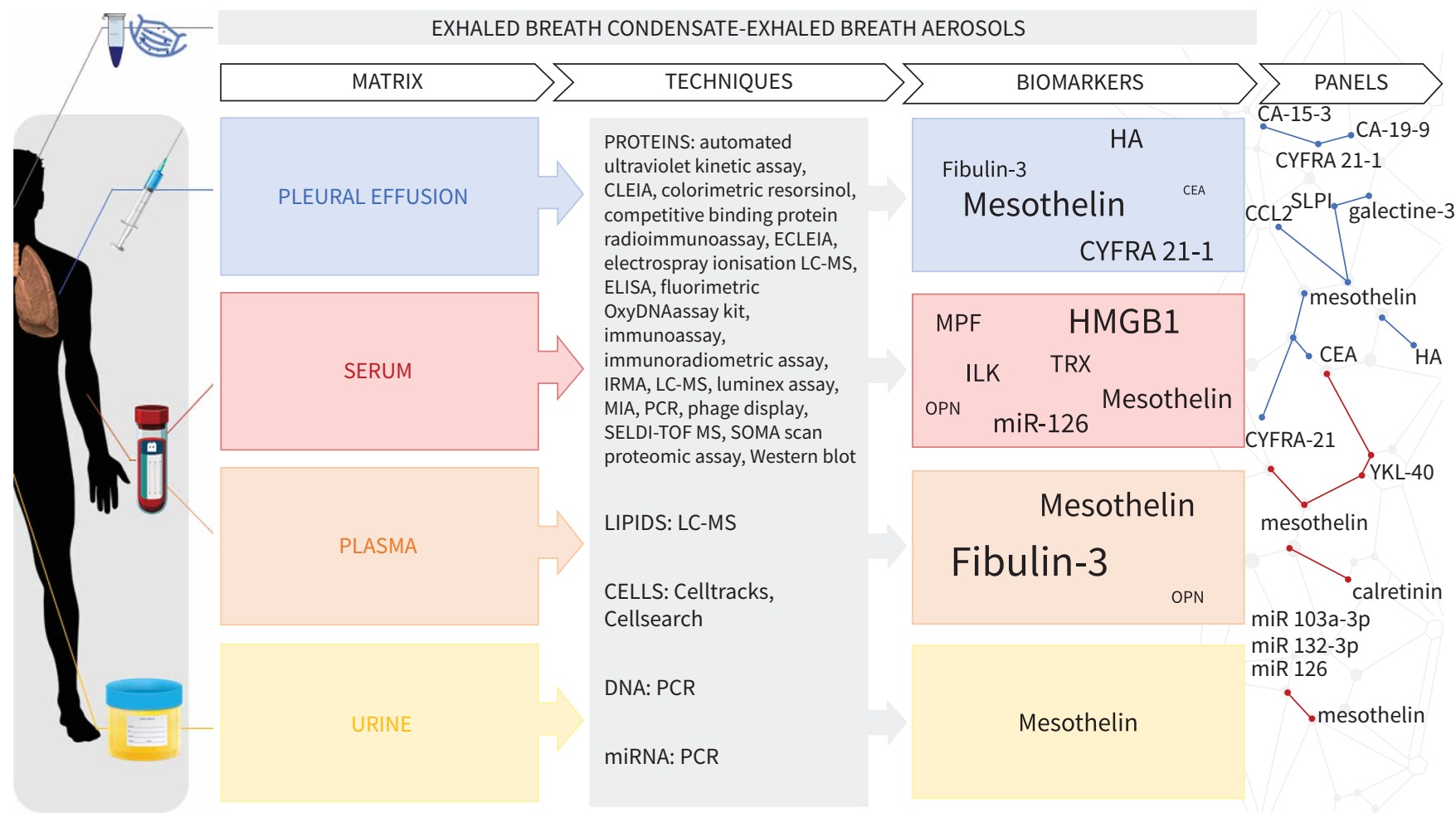

FIGURE 4 Liquid biomarkers for the diagnosis of malignant pleural mesothelioma (MPM). The font size of the different biomarkers is directly proportional to the area under the receiver operating characteristics curve ( $A \cup C_{R O C}$ ) obtained through the meta-analysis for that matrix. When detected in multiple matrices, the biomarker in that matrix resulting in the highest $A U C_{R O C}$ was put in bold. A few interesting combinations of biomarkers (biomarker panels), as discussed by articles included in this review, are shown in the figure under the column panel. Furthermore, this panel represents the possibility of combining different markers and matrices as a focus for future research. CEA: carcinoembryonic antigen; CLEIA: chemiluminescence immunoassay; CYFRA-21-1: cytokeratin 19 fragment; ECLEIA: electro-chemiluminescence immunoassay; ELISA: enzyme-linked immunosorbent assay; HA: hyaluronan; HMGB1: high mobility group box protein 1; ILK: integrin-linked protein kinase; IRMA: immunoradiometric assay; LC-MS: liquid chromatography-mass spectrometry; MIA: magnetic immunoassay; miR: microRNA; MPF: megakaryocyte potentiating factor; OPN: osteopontin; PCR: polymerase chain reaction; SELDI-TOF MS: surface-enhanced laser desorption/ionisation time of flight mass spectrometry; SLPI: secretory leukocyte protease inhibitor; TRX: thioredoxin; YKL: chitinase-like protein. 
between serum and PE mesothelin levels, with no significant favour. For OPN, the preferred matrix is plasma, as measurements have proven to be more stable and reliable. However, OPN was neither in plasma nor in serum the best-performing diagnostic marker [12]. This might be explained by its link to some other malignant conditions, as well as to non-malignant diseases such as tuberculosis [12]. In contrast, fibulin-3 has a clear superior diagnostic accuracy in plasma compared to PE. However, as the results of the included studies vary greatly, additional research is advised. Lastly, serum HA seems less than optimal due to the fast clearance from systemic circulation by stabilin-2 [3]. In pleural fluid, HA is present in the form of a large fibroblast-formed polysaccharide, making it more stable. Due to a lack in overlap between the miRNAs studied in the different matrices, no comparison of their performance between the matrices was possible. It is clear that it is impossible to just focus on one matrix, as different matrices are preferable for different markers (table 1; figure 4).

Mesothelin is by far the most investigated diagnostic biomarker for MPM. Despite repeated validation studies, there is no consensus yet about its actual clinical utility. A meta-analysis combining the diagnostic value of serum mesothelin in over 4000 patients resulted in $47 \%$ sensitivity at 95\% specificity [1, 89]. In this review, we obtained a summary $\mathrm{AUC}_{\mathrm{ROC}}$ of 0.81 for serum mesothelin when 27 studies were combined. Although promising, mesothelin clearly lacks the sensitivity to be used as standalone diagnostic marker. Therefore, focussing on a combination of different markers, either in one panel or using a decision tree, seems to be the best way forward. The combination of mesothelin, TRX and fibulin-3 in serum resulted in an optimised sensitivity and negative predictive value of $100 \%$ [29]. Although their specificities were disappointing, this $100 \%$ negative predictive value is ideal for a screening test, as all patients would be detected and referred for further (invasive) testing. The addition of biomarkers to the currently existing diagnostic techniques should also be further investigated. Adding PE mesothelin to cytological assessment reached a diagnostic sensitivity of $78.9 \%$, which is higher than the sensitivities of either individually [43]. Furthermore, we should be clear about what to expect from a diagnostic biomarker for malignant mesothelioma. Ideally, one strives for a perfect test, possessing a $100 \%$ sensitivity and $100 \%$ specificity. This holy grail test would lead to a flawless detection of MPM where we can perfectly distinguish MPM from all other diseases and no patients would be missed. However, a more realistic approach would be to either focus on a differential diagnosis with a high specificity or to focus on developing a test with a high sensitivity and negative predictive value, leading to a minimal of false negatives (supplementary figure S4). This test could then be implemented as a pre-diagnostic step for the AE population in the diagnosis of MPM. Due to its non-invasive nature, repeated testing is possible. Unfortunately, most current studies only included already diagnosed patients, with mostly advanced disease, which lack true clinical utility for the early stage markers and screening purposes. Future studies should therefore prospectively try and validate markers. This could prove to be difficult as several studies have indicated disease stage as a confounding factor, with earlier stages being more difficult to discriminate. PAss et al. [47] demonstrated this by separating stage I MPM from more advanced stages of MPM when compared to AE as control group. With an $\mathrm{AUC}_{\mathrm{ROC}}$ of 0.74 , stage I MPM was more difficult to discriminate from AE compared to advanced MPM (AUC ROC $=0.81$ ).

\begin{tabular}{|c|c|c|c|}
\hline & Serum & Plasma & PE \\
\hline Mesothelin & $0.81(0.79-0.83)$ & $0.86(0.79-0.92)$ & $0.83(0.81-0.85)$ \\
\hline MPF & $0.78(0.73-0.83)$ & & \\
\hline HMGB1 & $0.88(0.80-0.96)$ & & \\
\hline ILK & $0.79(0.59-0.99)$ & & \\
\hline OPN & $0.66(0.56-0.75)$ & $0.69(0.63-0.75)$ & \\
\hline TRX & $0.77(0.68-0.87)$ & & \\
\hline Fibulin3 & & $0.91(0.86-0.97)$ & $0.68(0.50-0.87)$ \\
\hline CEA & & & $0.55(0.21-0.89)$ \\
\hline CYFRA 21-1 & & & $0.73(0.67-0.78)$ \\
\hline HA & & & $0.78(0.73-0.82)$ \\
\hline $\operatorname{miR} 126$ & $0.80(0.68-0.91)$ & & \\
\hline
\end{tabular}

MPF: megakaryocyte potentiating factor; HMGB1: high-mobility group box 1; ILK: integrin-linked kinase; OPN osteopontin; PE: pleural effusion; TRX: thioredoxin; CEA: carcinoembryonic antigen; CYFRA 21-1: cytokeratin 19 fragments; HA: hyaluronan; miR: microRNA. The highest $A U C_{R O C}$ per matrix are in bold, the highest $A U C_{R O C}$ values per biomarker are underlined. 
Although no such studies were included in this review, a more recent and promising matrix is exhaled breath, more specifically the liquid phase enclosing exhaled breath condensate and aerosols. Both can be sampled using completely non-invasive techniques and contain a plethora of non-volatile or semi-volatile compounds, including, ammonia, isoprostanes, hydrogen peroxide, chemokines, cytokines, nitrogen oxides, peptides, DNA and RNA [90, 91]. By using a cooling device, exhaled breath is condensed into a liquid during tidal breathing. A rise in oxidative stress biomarkers in exhaled breath condensate has already been established in subjects with asbestosis compared to healthy controls [92]. Furthermore, promising results have been described regarding chronic airway diseases, such as cystic fibrosis, asthma and chronic obstructive pulmonary disease [93, 94]. Although the previously mentioned compounds are very diluted as breath also contains a lot of water, the matrix will still be less complex compared to serum or plasma. A more direct, but less explored approach is to entrap exhaled droplets, containing small non-volatile molecules, directly by the use of a filter or facemask. The origin of these aerosols can be linked to both the lower respiratory tract caused by surface film disruption and to the upper airways due to turbulence [91, 93, 95]. They are released during exhalation and in larger amounts during coughing and sneezing. However, caution is warranted not to mistake exogenous exposures for biomarker candidates. As every matrix has its own strengths and weaknesses, introducing a panel of optimal biomarkers beyond the borders of one matrix could have an added value.

\section{Conclusions and future perspectives}

We reviewed diagnostic liquid biomarkers for MPM. The majority focussed on identifying biomarkers in limited discovery cohorts, while implementation in clinical practice calls for larger validation studies. Furthermore, the heterogeneity of the used protocols and control groups was high, and efforts should therefore be made regarding standardisation to optimise results while making them comparable and reproducible. Focus should lie on the external validation of already discovered biomarkers, as well as exploring new biomarkers and biomarker panels. The most popular MPM biomarker is mesothelin and should be included in different panels, irrespective of matrix, as the combination with TRX and fibulin-3 has already shown promising results for screening purposes. Serum HMGB1 is also a strong contender, although further research is necessary to validate the positive results obtained by the two studies included in this review. The inclusion of patients with asbestosis or malignant diseases is encouraged as currently no difference in HMGB1 levels was found between asbestosis and MPM. Based on this review, plasma fibulin-3 $\left(\mathrm{AUC}_{\mathrm{ROC}}=0.91\right)$ is the most promising single biomarker and should therefore be put in the limelight when further research is concerned. Furthermore, the use of new and innovative technologies for panel discovery should be encouraged. As MPM is mostly diagnosed at an advanced stage, the discovery and validation of early stage biomarkers is lacking. The set-up of an overarching biobank of early stage samples for scientific use could lead to a boost in this specific type of research. Furthermore, as different liquid matrices provide matrix-specific biomarkers, the combination of biomarkers from different matrices should be further investigated, as they each have their different strengths and downfalls. Lastly, recent discoveries in exhaled breath condensate and aerosols should be explored.

Provenance: Submitted article, peer reviewed.

Author contributions: E. Schillebeeckx: data curation, investigation, methodology, visualisation, writing (original draft, review and editing). J.P. van Meerbeeck: supervision, writing (review and editing). K. Lamote: conceptualisation, methodology, funding acquisition, supervision, visualisation, writing (review and editing).

Conflict of interest: None declared.

Support statement: This study was funded by a grant from the Foundation Against Cancer (grant STK 2018-137) and by a grant from Kom op tegen Kanker (Stand up to Cancer), the Flemish cancer society by the grant "Emmanuel van der Schueren". Funding information for this article has been deposited with the Crossref Funder Registry.

\section{References}

1 Scherpereel A, Opitz I, Berghmans T, et al. ERS/ESTS/EACTS/ESTRO guidelines for the management of malignant pleural mesothelioma. Eur Respir J 2020; 55: 1900953.

2 Delgermaa V, Takahashi K, Park EK, et al. Global mesothelioma deaths reported to the World Health Organization between 1994 and 2008. Bull World Health Organ 2011; 89: 716-724, 724A-724C.

3 Arnold DT, Maskell NA. Biomarkers in mesothelioma. Ann Clin Biochem 2018; 55: 49-58.

4 Lamote K, Nackaerts K, van Meerbeeck JP. Strengths, weaknesses, and opportunities of diagnostic breathomics in pleural mesothelioma-a hypothesis. Cancer Epidemiol Biomarkers Prev 2014; 23: 898-908. 
5 Robinson BWS, Creaney J, Lake R, et al. Soluble mesothelin-related protein - a blood test for mesothelioma. Lung Cancer 2005; 49: S109-S111.

6 Baas P, Scherpereel A, Nowak AK, et al. First-line nivolumab plus ipilimumab in unresectable malignant pleural mesothelioma (CheckMate 743): a multicentre, randomised, open-label, phase 3 trial. Lancet 2021; 397: 375-386.

7 Bardelli A, Pantel K. Liquid biopsies, what we do not know (yet). Cancer Cell 2017; 31: 172-179.

8 Bai Y, Zhao H. Liquid biopsy in tumors: opportunities and challenges. Ann Transl Med 2018; 6: Suppl. 1, S89.

9 Moher D, Liberati A, Tetzlaff J, et al. Preferred reporting items for systematic reviews and meta-analyses: the PRISMA statement. PLoS Med 2009; 6: e1000097.

10 Whiting PF, Rutjes AW, Westwood ME, et al. QUADAS-2: a revised tool for the quality assessment of diagnostic accuracy studies. Ann Intern Med 2011; 155: 529-536.

11 Viechtbauer W. Conducting meta-analyses in R with the metafor package. J Stat Softw 2010; 36: i02.

12 Cristaudo A, Foddis R, Bonotti A, et al. Comparison between plasma and serum osteopontin levels: usefulness in diagnosis of epithelial malignant pleural mesothelioma. Int J Biol Markers 2010; 25: 164-170.

13 Cristaudo A, Bonotti A, Simonini S, et al. Combined serum mesothelin and plasma osteopontin measurements in malignant pleural mesothelioma. J Thorac Oncol 2011; 6: 1587-1593.

14 Bonotti A, Foddis R, Landi S, et al. A novel panel of serum biomarkers for MPM diagnosis. Dis Markers 2017; 2017: 3510984.

15 Cristaudo A, Foddis R, Bonotti A, et al. Two novel polymorphisms in 5' flanking region of the mesothelin gene are associated with soluble mesothelin-related peptide (SMRP) levels. Int J Biol Markers 2011; 26: 117-123.

16 Jiang ZQ, Ying SB, Shen W, et al. Plasma fibulin-3 as a potential biomarker for patients with asbestos-related diseases in the Han population. Dis Markers 2017; 8: 1725354.

17 Cappellesso R, Nicole L, Caroccia B, et al. Young investigator challenge: MicroRNA-21/MicroRNA-126 profiling as a novel tool for the diagnosis of malignant mesothelioma in pleural effusion cytology. Cancer Cytopathol 2016; 124: 28-37.

18 Santarelli L, Strafella E, Staffolani S, et al. Association of MiR-126 with soluble mesothelin-related peptides, a marker for malignant mesothelioma. PLOS One 2011; 6: 9.

19 Tomasetti M, Staffolani S, Nocchi L, et al. Clinical significance of circulating miR-126 quantification in malignant mesothelioma patients. Clin Biochem 2012; 45: 575-581.

20 Scherpereel A, Grigoriu B, Conti M, et al. Soluble mesothelin-related peptides in the diagnosis of malignant pleural mesothelioma. Am J Respir Crit Care Med 2006; 173: 1155-1160.

21 Grigoriu BD, Scherpereel A, Devos P, et al. Utility of osteopontin and serum mesothelin in malignant pleural mesothelioma diagnosis and prognosis assessment. Clin Cancer Res 2007; 13: 2928-2935.

22 Paleari L, Rotolo N, Imperatori A, et al. Osteopontin is not a specific marker in malignant pleural mesothelioma. Int J Biol Markers 2009; 24: 112-117.

23 Amati M, Tomasetti M, Scartozzi M, et al. Profiling tumor-associated markers for early detection of malignant mesothelioma: an epidemiologic study. Cancer Epidemiol Biomarkers Prev 2008; 17: 163-170.

24 Matboli M, Shafei AE, Azazy AEM, et al. Clinical evaluation of circulating miR-548a-3p and-20a expression in malignant pleural mesothelioma patients. Biomark Med 2018; 12: 129-139.

25 Muley $\mathrm{T}$, Dienemann $\mathrm{H}$, Herth FJF, et al. Combination of mesothelin and CEA significantly improves the differentiation between malignant pleural mesothelioma, benign asbestos disease, and lung cancer. $J$ Thorac Oncol 2013; 8: 947-951.

26 Dongel I, Akbas A, Benli I, et al. Comparison of serum biochemical markers in patients with mesothelioma and pleural plaques versus healthy individuals exposed to environmental asbestos. Turk Gogus Kalp Damar Cerrahisi Derg 2019; 27: 374-380.

27 Alatas F, Alatas O, Metintas M, et al. Diagnostic value of CEA, CA 15-3, CA 19-9, CYFRA 21-1, NSE and TSA assay in pleural effusions. Lung Cancer 2001; 31: 9-16.

28 Sato $\mathrm{H}$, Soh J, Aoe K, et al. Droplet digital PCR as a novel system for the detection of microRNA-34b/c methylation in circulating DNA in malignant pleural mesothelioma. Int J Oncol 2019; 54: 2139-2148.

29 Demir M, Kaya H, Taylan M, et al. Evaluation of new biomarkers in the prediction of malignant mesothelioma in subjects with environmental asbestos exposure. Lung 2016; 194: 409-417.

30 Holdenrieder S, Hatz R, Reinmiedl J, et al. Improved diagnosis of mesothelioma by a combination of soluble mesothelin-related peptide and CYFRA 21-1. Laboratoriums Medizin 2015; 39: 103-113.

31 Bayram M, Dongel I, Akbas A, et al. Serum biomarkers in patients with mesothelioma and pleural plaques and healthy subjects exposed to naturally occurring asbestos. Lung 2014; 192: 197-203.

32 Suzuki H, Hirashima T, Kobayashi M, et al. Cytokeratin 19 fragment/carcinoembryonic antigen ratio in pleural effusion is a useful marker for detecting malignant pleural mesothelioma. Anticancer Res 2010; 30: 4343-4346.

33 Mundt F, Johansson HJ, Forshed J, et al. Proteome screening of pleural effusions identifies galectin 1 as a diagnostic biomarker and highlights several prognostic biomarkers for malignant mesothelioma. Mol Cell Proteomics 2014; 13: 701-715. 
34 Arslan S, Mundt F, Metintas S, et al. Predicting malignant mesothelioma by analyzing serum N-ERC/mesothelin, C-ERC/mesothelin, hyaluronan, osteopontin, and syndecan-1 levels. Eurasian J Pulmonol 2017; 19: 130-138.

35 Hooper CE, Morley AJ, Virgo P, et al. A prospective trial evaluating the role of mesothelin in undiagnosed pleural effusions. Eur Respir J 2013; 41: 18-24.

36 Wang XF, Wu YH, Wang MS, et al. CEA, AFP, CA125, CA153 and CA199 in malignant pleural effusions predict the cause. Asian Pac J Cancer Prev 2014; 15: 363-368.

37 Mundt F, Nilsonne G, Arslan S, et al. Hyaluronan and N-ERC/mesothelin as key biomarkers in a specific two-step model to predict pleural malignant mesothelioma. PLOS One 2013; 8: e72030.

38 Cavallari I, Urso L, Sharova E, et al. Liquid biopsy in malignant pleural mesothelioma: state of the art, pitfalls, and perspectives. Front Oncol 2019; 9: 740.

39 Dipalma N, Luisi V, Di Serio F, et al. Biomarkers in malignant mesothelioma: diagnostic and prognostic role of soluble mesothelin-related peptide. Int J Biol Markers 2011; 26: 160-165.

40 Cristaudo A, Foddis R, Vivaldi A, et al. Clinical significance of serum mesothelin in patients with mesothelioma and lung cancer. Clin Cancer Res 2007; 13: 5076-5081.

41 Schneider J, Hoffmann H, Dienemann H, et al. Diagnostic and prognostic value of soluble mesothelin-related proteins in patients with malignant pleural mesothelioma in comparison with benign asbestosis and lung cancer. J Thorac Oncol 2008; 3: 1317-1324.

42 Beyer HL, Geschwindt RD, Glover CL, et al. MESOMARK (TM): A potential test for malignant pleural mesothelioma. Clin Chem 2007; 53: 666-672.

43 Franceschini MC, Ferro P, Canessa PA, et al. Mesothelin in serum and pleural effusion in the diagnosis of malignant pleural mesothelioma with non-positive cytology. Anticancer Res 2014; 34: 7425-7429.

44 Ferro P, Canessa PA, Battolla E, et al. Mesothelin is more useful in pleural effusion than in serum in the diagnosis of pleural mesothelioma. Anticancer Res 2013; 33: 2707-2713.

45 Portal JAR, Becerra ER, Rodriguez DR, et al. Serum levels of soluble mesothelin-related peptides in malignant and nonmalignant asbestos-related pleural disease: relation with past asbestos exposure. Cancer Epidemiol Biomarkers Prev 2009; 18: 646-650.

46 Rai AJ, Flores RM, Mathew A, et al. Soluble mesothelin related peptides (SMRP) and osteopontin as protein biomarkers for malignant mesothelioma: analytical validation of ELISA based assays and characterization at mRNA and protein levels. Clin Chem Lab Med 2010; 48: 271-278.

47 Pass HI, Wali A, Tang N, et al. Soluble mesothelin-related peptide level elevation in mesothelioma serum and pleural effusions. Ann Thorac Surg 2008; 85: 265-272.

48 Santarelli L, Staffolani S, Strafella E, et al. Combined circulating epigenetic markers to improve mesothelin performance in the diagnosis of malignant mesothelioma. Lung Cancer 2015; 90: 457-464.

49 Napolitano A, Antoine DJ, Pellegrini L, et al. HMGB1 and its hyperacetylated isoform are sensitive and specific serum biomarkers to detect asbestos exposure and to identify mesothelioma patients. Clin Cancer Res 2016; 22: 3087-3096.

50 Creaney J, Yeoman D, Demelker Y, et al. Comparison of osteopontin, megakaryocyte potentiating factor, and mesothelin proteins as markers in the serum of patients with malignant mesothelioma. $J$ Thorac Oncol 2008; 3: 851-857.

51 Ak G, Tada $\mathrm{Y}$, Shimada $\mathrm{H}$, et al. Midkine is a potential novel marker for malignant mesothelioma with different prognostic and diagnostic values from mesothelin. BMC Cancer 2017; 17: 10.

52 Creaney J, Musk AW, Robinson BWS. Sensitivity of urinary mesothelin in patients with malignant mesothelioma. J Thorac Oncol 2010; 5: 1461-1466.

53 Johnen G, Gawrych K, Raiko I, et al. Calretinin as a blood-based biomarker for mesothelioma. BMC Cancer 2017; 17: 12.

54 Creaney J, Yeoman D, Naumoff LK, et al. Soluble mesothelin in effusions: a useful tool for the diagnosis of malignant mesothelioma. Thorax 2007; 62: 569-576.

55 Creaney J, Yeoman D, Musk AW, et al. Plasma versus serum levels of osteopontin and mesothelin in patients with malignant mesothelioma-which is best? Lung Cancer 2011; 74: 55-60.

56 Creaney J, Sneddon S, Dick IM, et al. Comparison of the diagnostic accuracy of the MSLN gene products, mesothelin and megakaryocyte potentiating factor, as biomarkers for mesothelioma in pleural effusions and serum. Dis Markers 2013; 35: 119-127.

57 Sriram KB, Relan V, Clarke BE, et al. Pleural fluid cell-free DNA integrity index to identify cytologically negative malignant pleural effusions including mesotheliomas. BMC Cancer 2012; 12: 428.

58 Corradi M, Goldoni M, Alinovi R, et al. YKL-40 and mesothelin in the blood of patients with malignant mesothelioma, lung cancer and asbestosis. Anticancer Res 2013; 33: 5517-5524.

59 Hollevoet K, Nackaerts K, Thimpont J, et al. Diagnostic performance of soluble mesothelin and megakaryocyte potentiating factor in mesothelioma. Am J Respir Crit Care Med 2010; 181: 620-625.

60 Fujimoto N, Ohnuma K, Aoe K, et al. Clinical significance of soluble CD26 in malignant pleural mesothelioma. PLOS One 2014; 9: 14. 
61 Creaney J, van Bruggen I, Hof M, et al. Combined CA125 and mesothelin levels for the diagnosis of malignant mesothelioma. Chest 2007; 132: 1239-1246.

62 Pass HI, Lott D, Lonardo F, et al. Asbestos exposure, pleural mesothelioma, and serum osteopontin levels. N Engl J Med 2005; 353: 1564-1573.

63 Watzka SB, Posch F, Pass HI, et al. Detection of integrin-linked kinase in the serum of patients with malignant pleural mesothelioma. J Thorac Cardiovasc Surg 2011; 142: 384-389.

64 Watzka SB, Posch F, Pass HI, et al. Serum concentration of integrin-linked kinase in malignant pleural mesothelioma and after asbestos exposure. Eur J Cardio-Thorac Surg 2013; 43: 940-944.

65 Maeda R, Tabata C, Tabata R, et al. Is Serum thioredoxin-1 a useful clinical marker for malignant pleural mesothelioma? Antioxid Redox Signal 2011; 15: 685-689.

66 Ying SB, Jiang ZQ, He XL, et al. Serum HMGB1 as a potential biomarker for patients with asbestos-related diseases. Dis Markers 2017; 9: 5756102.

67 Creaney J, Dick IM, Meniawy TM, et al. Comparison of fibulin-3 and mesothelin as markers in malignant mesothelioma. Thorax 2014; 69: 895-902.

68 Pass HI, Levin SM, Harbut MR, et al. Fibulin-3 as a blood and effusion biomarker for pleural mesothelioma. N Engl J Med 2012; 367: 1417-1427.

69 Johnen G, Burek K, Raiko I, et al. Prediagnostic detection of mesothelioma by circulating calretinin and mesothelin - a case-control comparison nested into a prospective cohort of asbestos-exposed workers. Sci Rep 2018; 8: 11.

70 Jimenez-Ramirez C, Casjens S, Juarez-Perez CA, et al. Mesothelin, calretinin, and megakaryocyte potentiating factor as biomarkers of malignant pleural mesothelioma. Lung 2019; 197: 641-649.

71 Weber DG, Casjens S, Johnen G, et al. Combination of MiR-103a-3p and mesothelin improves the biomarker performance of malignant mesothelioma diagnosis. PLOS One 2014; 9: 15.

72 Weber DG, Gawrych K, Casjens S, et al. Circulating miR-132-3p as a candidate diagnostic biomarker for malignant mesothelioma. Dis Markers 2017; 15: 9280170.

73 Davies HE, Sadler RS, Bielsa S, et al. Clinical impact and reliability of pleural fluid mesothelin in undiagnosed pleural effusions. Am J Respir Crit Care Med 2009; 180: 437-444.

74 Creaney J, Segal A, Olsen N, et al. Pleural fluid mesothelin as an adjunct to the diagnosis of pleural malignant mesothelioma. Dis Markers 2014; 2014: 413946.

75 Canessa PA, Franceschini MC, Ferro P, et al. Evaluation of soluble mesothelin-related peptide as a diagnostic marker of malignant pleural mesothelioma effusions: its contribution to cytology. Cancer Invest 2013; 31: 43-50.

76 Aleman C, Porcel JM, Segura RM, et al. Pleural fluid mesothelin for the differential diagnosis of exudative pleural effusions. Med Clin 2009; 133: 449-453.

77 Fujimoto N, Gemba K, Asano M, et al. Soluble mesothelin-related protein in pleural effusion from patients with malignant pleural mesothelioma. Exp Ther Med 2010; 1: 313-317.

78 Battolla E, Canessa PA, Ferro P, et al. Comparison of the diagnostic performance of Fibulin-3 and mesothelin in patients with pleural effusions from malignant mesothelioma. Anticancer Res 2017; 37: 1387-1391.

79 Blanquart C, Gueugnon F, Nguyen JM, et al. CCL2, galectin-3, and SMRP combination improves the diagnosis of mesothelioma in pleural effusions. J Thorac Oncol 2012; 7: 883-889.

80 Filiberti R, Parodi S, Libener R, et al. Diagnostic value of mesothelin in pleural fluids: comparison with CYFRA 21-1 and CEA. Med Oncol 2013; 30: 543.

81 Otoshi T, Kataoka Y, Ikegaki S, et al. Pleural effusion biomarkers and computed tomography findings in diagnosing malignant pleural mesothelioma: a retrospective study in a single center. PLOS One 2017; 12: e0185850.

82 Hegmans J, Veltman JD, Fung ET, et al. Protein profiling of pleural effusions to identify malignant pleural mesothelioma using SELDI-TOF MS. Technol Cancer Res Treat 2009; 8: 323-332.

83 Fuhrman C, Duche JC, Chouaid C, et al. Use of tumor markers for differential diagnosis of mesothelioma and secondary pleural malignancies. Clin Biochem 2000; 33: 405-410.

84 Bray BA. Chapter 11 - hyaluronan in the pulmonary alveolus and interstitium. In: Garg HG, Hales CA, eds. Chemistry and Biology of Hyaluronan. Oxford, Elsevier Science Ltd, 2004; pp. 247-269.

85 Birnie KA, Prele CM, Musk AW, et al. MicroRNA signatures in malignant pleural mesothelioma effusions. Dis Markers 2019; 2019: 9.

86 Ostroff RM, Mehan MR, Stewart A, et al. Early detection of malignant pleural mesothelioma in asbestos-exposed individuals with a noninvasive proteomics-based surveillance tool. PLOS One 2012; 7: 10.

87 Weber DG, Johnen G, Bryk O, et al. Identification of miRNA-103 in the cellular fraction of human peripheral blood as a potential biomarker for malignant mesothelioma - a pilot study. PLOS One 2012; 7: 9.

88 Hollevoet K, Nackaerts K, Thas $\mathrm{O}$, et al. The effect of clinical covariates on the diagnostic and prognostic value of soluble mesothelin and megakaryocyte potentiating factor. Chest 2012; 141: 477-484.

89 Hollevoet K, Reitsma JB, Creaney J, et al. Serum mesothelin for diagnosing malignant pleural mesothelioma: an individual patient data meta-analysis. J Clin Oncol 2012; 30: 1541-1549. 
90 Brusselmans L, Arnouts L, Millevert C, et al. Breath analysis as a diagnostic and screening tool for malignant pleural mesothelioma: a systematic review. Transl Lung Cancer Res 2018; 7: 520-536.

91 Wallace MAG, Pleil JD. Evolution of clinical and environmental health applications of exhaled breath research: Review of methods and instrumentation for gas-phase, condensate, and aerosols. Anal Chim Acta 2018; 1024: 18-38.

92 Chow S, Campbell C, Sandrini A, et al. Exhaled breath condensate biomarkers in asbestos-related lung disorders. Respir Med 2009; 103: 1091-1097.

93 Konstantinidi EM, Lappas AS, Tzortzi AS, et al. Exhaled breath condensate: technical and diagnostic aspects. Scientific World J 2015; 2015: 435160.

94 Horváth I, Hunt J, Barnes PJ. Exhaled breath condensate: methodological recommendations and unresolved questions. Eur Respir J 2005; 26: 523.

95 Pleil JD, Beauchamp JD, Risby TH, et al. The scientific rationale for the use of simple masks or improvised facial coverings to trap exhaled aerosols and possibly reduce the breathborne spread of COVID-19. $J$ Breath Res 2020; 14: 030201. 